\title{
PROCESS AND SYSTEMS Learning from critical care: Improving intra- and inter-hospital transfer processes in enhanced care and the ward
}

\author{
Authors: Kathleen Bonnici, ${ }^{\mathrm{A}}$ Richard Ridings, ${ }^{\mathrm{B}}$ Roger Chinn, ${ }^{\mathrm{C}}$ Gezz Van Zwanenberg, ${ }^{\mathrm{D}}$ Angela Walsh, \\ Timothy Wigmore, ${ }^{F}$ Maxine Wilkie ${ }^{G}$ and Gary Davies ${ }^{H}$
}

The Intensive Care Society (ICS) has recently published guidance on the transfer of critically ill adults. Since 2007, the North West London Critical Care Network has documented and audited patient transfers undertaken across 13 hospitals, and trained staff in transferring critically ill or injured patients. In 2015/16 the network introduced intra-hospital transfer documentation and applied the same transfer training and audit methodology for critically ill patients being moved within hospitals. While increasing data capture and with a targeted training strategy adapted from transfer experience, the network has seen a reduction in number and severity of incidents in the region.

In contrast to this experience, no formalised processes exists to support the inter-hospital or intra-hospital transfer of patients from enhanced care areas or wards not embedded within critical care. Often very little data is collected from these areas, but we suspect transfer risks are higher in these cases as a consequence of the deficit of structured transfers. In collaboration with members of the critical care network, we have developed a basic training module along with the use of a transfer form which has been well received and had a positive impact in terms of incidents reported.

KEYWORDS: Intra-hospital transfer, inter-hospital transfer, critical care, medical wards, checklist

DOI: 10.7861/fhj.2019-0063

Authors: ${ }^{\mathrm{A}}$ consultant in acute and general internal medicine, Chelsea and Westminster Hospital, London, UK; ${ }^{B}$ acute medicine practice development nurse, Chelsea and Westminster Hospital, London, UK; C trust medical director (acting), Chelsea and

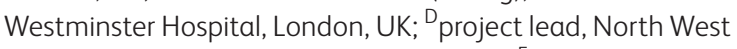
London Critical Care Network, London, UK; ${ }^{E}$ director, North West London Critical Care Network, London, UK; ${ }^{F}$ consultant in intensive care medicine and chair, North West London Critical Care Network, London, UK; ${ }^{G}$ acute care common stem trainee, Chelsea and Westminster Hospital, London, UK; ${ }^{H}$ consultant in respiratory and acute medicine and hospital medical director, Chelsea and Westminster Hospital, London, UK

\section{Introduction}

Robust transfer standards exist for the sickest of patients in our hospitals, often with the support of local critical care networks.' The North West London Critical Care Network developed guidelines and documentation that act to help prompt the clinician and also functions as an audit tool to help improve safety and consistency in the standards of the transfer of critically unwell patients. Transfer training is now incorporated as a standard part of network-wide training programmes for nurses, doctors and some allied healthcare professionals working with critical care patients. This provides further support to many of the standards put forward by the London Health Programmes' 2014 inter-hospital transfer standards. ${ }^{1}$ Since 2007, the network has collected data from all critical care transfers in north-west London. There is evidence that, over time, there has been a fall in incidents (both intra- and inter-hospital; Fig 1) which is likely to be associated with the dissemination of transfer training to critical care nurses and doctors by the network and other bodies, as well as the increased use of checklists. ${ }^{2}$

Features of the processes used include standardised patient transfer documentation, risk assessment, access to free transfer training for staff, development of a transfer app (application for a smart phone), standardised emergency equipment, and use of a standardised network transfer bag in each hospital and each area from which critical care patient transfers arise.

The network's experience and audit data confirm that many trusts, including our own, do not have such consistent processes as standard when transferring our enhanced care patients (level 1 or enhanced care) to areas such as radiology or from one hospital to another. Arguably, the least safe transfers now occurring are those for deteriorating ward patients who have not yet undergone stabilisation. While some will have been correctly identified and escalated to the intensive care unit and/or outreach teams to have input in the patient's care and the adoption of a standardised transfer process as required, we were concerned that there were many patients for whom this was not the case. As part of this work, we looked what was already available from the critical care network and to see what could be adapted for deteriorating ward or enhanced care patients.

\section{The problem: The 'risky' ward transfer}

An incident after the transfer of a ward medical patient in 2017 prompted the review of transfer processes for enhanced care area 



Fig 1. Hospital critical care patient transfers in north-west London 2007-2019. a) Incident rate in transfers between hospitals (inter-hospital). b) Incident rate in transfers within hospitals (intrahospital). ${ }^{2}$ patients at Chelsea and Westminster Hospital NHS Foundation Trust. This revealed two incidents that occurred on transfer from the ward to radiology and it appeared that the risks of intrahospital transfer were underestimated, most likely because of familiarity with the surroundings and perception that they are quick and involve short distances. Despite the risks being often as great as an inter-hospital transfer, there is often much less planning, preparation or thought about handover process and it may be left to junior members of staff who have had no formal teaching on transfer safety. This arises from poor understanding of the risks involved, staff pressures and lack of formal teaching on the process, which increases the hazardous nature of the transfer. This was backed up by perceptions of teachers on network courses who felt that staff were more likely to use checklists etc if there was an ambulance journey.

We found handover processes were variable, with inconsistent and incomplete documentation for both intra- and inter-hospital ward transfers. This occurred concurrently with increasing numbers of transfers of patients for diagnostic and interventional procedures since the expansion of the trust to cover two sites.

In contrast to critical care staff, ward nursing and medical staff have often not received any formal transfer training. Such training would usually include the planning of equipment, transport, staffing seniority and methodology of handover processes, and we felt that lessons such as these could be learnt from our critical care colleagues. Many studies have shown that the majority of problems observed during transfers are preventable by adequate pre-communication and planning, and also that the numbers of incidents are likely to be underestimated. ${ }^{3,4}$

\section{Example of an intervention}

The acute medical team collaborated with the critical care network and developed our own transfer training process for both intraand inter-hospital transfers, focusing on the level 1 and enhanced care patients that would typically be in our ward 'enhanced care area'. This area is staffed by non-critical care trained staff, none of whom had previously undergone critical care transfer training. Starting in February 2019, we trained 95 nursing and medical staff (only one had had any transfer training previously) and began a 4-monthly cycle of training for those staff coming through the ward area. We drew attention to Intensive Care Society (ICS) guideline recommendation 17 that states that a similar process should be followed whether the patient is being moved within or to another hospital. ${ }^{5}$

We were keen to provide training at an appropriate level for ward nursing and medical staff who may have had no critical care experience and for a patient population receiving much less support than those in critical care. However, we agreed with the 


\section{Box 1. Key parts of initiative}

Presentation including case study discussion, transfer app, physiological effects of transfer and how to fill out the transfer form

Situation, background, assessment and recommendation (SBAR) method of handove

Practical training of portable oxygen and suction

Transfer form that is now evaluated monthly on number and quality

New transfer bag

themes recommended by the ICS guidelines that suggest dividing your preparation into patient, staff, equipment, organisation and departure. ${ }^{5}$ Many sections would naturally be briefer for the ward patient and there would, therefore, be focus on factors such as the amount of oxygen the patient required for transfer, the means of delivery and anticipation of steps needed should the patient deteriorate. We utilised the adult patient escort risk assessment tool and found that while many doctors (even at registrar level) were unaware of it, they subsequently found it very useful (supplementary material S1). Having reviewed the network standard transfer bag, rather than the advanced resuscitation tools we developed a simple enhanced care transfer bag with fluids, intravenous access and basic airway adjuncts, and the bag valve mask in order to match the skills of the escorting team and the likely requirements of the patients.

We adapted existing network intra-hospital documentation (already used in the emergency department and ICU) to develop an appropriate accompanying transfer form (supplementary material S2). ${ }^{6}$ This acts as a prompt for current issues including diagnosis, National Early Warning Score (NEWS), the resuscitation status, equipment checklist and provides space for recording observations during transfer and time off the ward (Box 1).

In our observation, we found that the teaching given to junior doctors and nurses in how to undertake handovers was frequently poor. We therefore put a great deal of prominence on this aspect of training to ensure that there was a much greater awareness of the required verbal and written handover standards for transfers between department, hospital site or between institutions. ${ }^{1,5} \mathrm{An}$ SBAR (situation, background, assessment and recommendation) model was adopted with emphasis on keeping all necessary parties updated on both the impending transfer and on the situation of the patient to prevent unanticipated arrival of a patient or lack of awareness of possible care requirements at the recipient site with resultant increased risk.

In the 8 months since the introduction of the training programme and the simple tools such as the pro forma, there have been no further incidents reported concerning enhanced care / ward patient transfers at the acute assessment unit of the Chelsea site where this has been rolled out. Those that went through the training were surveyed and $87 \%$ found it very or extremely relevant to their jobs, and many commented that they now felt more confident in undertaking patient transfers. An audit of forms has shown that 133 forms have been used to date on our nine-bedded unit (the majority of these were to radiology and endoscopy).

\section{Discussion}

We have shared our experience of improvement of transfer processes for non-critical care patients using a training programme and a transfer form that we developed from experience gained within our critical care network. The network team had a key role in training our nursing and medical staff.

In summary, we feel lessons can be learnt from adopting dedicated transfer training for patients in enhanced care areas (level 1 or enhanced care) in a similar manner to that proposed by the ICS, National Competency Framework for Registered Practitioners: Level 1 Patients and Enhanced Care Areas and also The Faculty of Intensive Care Medicine working party document (points 3.2.2 and 4.2.2). ${ }^{7,8}$ Adopting a robust governance process seems to have reduced incidents on our acute assessment unit, better handovers and documentation, and greater confidence within the nursing and medical staff. When such patients are put in a cohort within a level 1 or enhanced care area, the training of medical and nursing staff is made much easier. However, identification of these patients in other care environments via NEWS or other risk assessment tools can help to ensure that appropriate measures are taken whenever they are transferred.

In a process analogous to that which has happened over the last decade in critical care, we propose the adoption, dissemination and evaluation of standardised transfer methods and tools when looking after unwell ward patients. Ideally the training and unification of processes across trusts will serve to safeguard against the risks of patients deteriorating during transfers and to better prepare teams should such deteriorations occur.

\section{Supplementary material}

Additional supplementary material may be found in the online version of this article at www.rcpjournals.org/fhj:

S1 - Risk assessment tool for minimum escort requirements.

S2 - Intra-hospital transfer form.

\section{References}

1 London Health Programmes. Quality and safety programme: Interhopsital transfers - adults. London Health Programmes, 2014. www.londonccn.nhs.uk/media/1586/final-adult-iht-standards updated.pdf

2 North West London Critical Care Network. Annual Report 20182019. North West London Critical Care Network.

3 Grier S, Brant G, Gould TH et al. Critical care transfer in an English critical care network: Analysis of 1124 transfers delivered by an ad-hoc system. J Intensive Care Soc 2020;21:33-9.

4 Lovell MA, Mudaliar MY, Klineberg PL. Intrahospital transfer of critically ill patients: complications and difficulties. Anaesth Intensive Care 2001;29:400-5.

5 Intensive Care Society, The Faculty of Intensive Care Medicine. Guidance on: the transfer of the critically ill adult. ICS, 2019.

6 Dransfield M. Intra hospital transfer form: level 2 or 3 patients. North West London Critical Care Network, 2015. www.londoncen. nhs.uk/managing-the-unit/patient-transfer/intra-hospital-withinhospital-transfers

7 National Outreach Forum, Critical Care Networks - National Nurse Leads. National Competency Framework for Registered Practitioners: Level 1 Patients and Enhanced Care Areas. The Faculty of Intensive Care Medicine, 2018:62-3. www.ficm.ac.uk/ sites/default/files/level_1_competencies_final_7.7.18.pdf 
8 The Faculty of Intensive Care Medicine. Enhanced care: guidance on service development in acute care. FICM, 2019:8,10. www.ficm. ac.uk/sites/default/files/enhanced_care_guidance_for_open_ consultation_nov_2019_v2.pdf
Address for correspondence: Dr Kathleen Bonnici, Chelsea and Westminster Hospital, 369 Fulham Road, London SW10 9NH, UK.

Email: kathleen.bonnici@chelwest.nhs.uk

\title{
Management and care of tracheostomised patients with prolonged disorders of consciousness (PDOC) during COVID-19
}

\begin{abstract}
This guidance, which supplements the PDOC national clinical guidelines published in March 2020, supports decision-making in tracheostomised patients with PDOC and takes into account the requirements to protect patients and staff from the risks of acquiring COVID-19.
\end{abstract}

Download the guidelines: www.rcplondon.ac.uk/pdoc-covid-19 